\title{
Formas de Gobierno Municipal y de Elecciones Locales en Estados Unidos y Colombia
}

\section{Municipal forms of government and of local elections in the United States and Colombia}

\author{
AUGUSTO HERNÁNDEZ VIDAL \\ Egresado Facultad de Derecho de la Universidad Santo Tomás Bogotá. Correo \\ electrónico: augustohernandezvidal@hotmail.com \\ Graduate from the Law Faculty of the Santo Tomas University, Bogota. E-mail: \\ augustohernandezvidal@hotmail.com
}

Fecha de recepción: 20 de octubre de 2010 Fecha de evaluación: 28 de octubre de 2010 Fecha de aprobación: 30 de mayo de 2011

RESUMEN: La legislación colombiana en materia de estructura y administración pública se ha pensado para las grandes ciudades, que cuentan con recursos físicos y humanos avanzados, para desempeñar las funciones administrativas más complejas, más tecnológicas y más ritualistas. En las pequeñas poblaciones colombianas las autoridades y los funcionarios deben organizarse y ejercer sus labores de acuerdo a esas normas, que no han tomado en cuenta las particularidades de los municipios medianos y pequeños. Una reflexión sincera del tema no deja más opciones que otorgar a los municipios que se encuentran en situaciones especiales la facultad para elegir una organización y una estructura razonable a sus necesidades. En este artículo se toma como reseña la experiencia estadounidense, donde cada Estado tiene la facultad legal para ofrecer formas de 
gobierno diversas a sus municipios y condados, cada una con sistemas electorales particulares.

PALABRAS CLAVE: Formas de gobierno local, sistemas electorales, métodos de nominación, uniformidad estructural, democracia local.

SUMMARY: Colombian legislation on structure and public administration is usually written for large cities that have the physical and human resources needed to manage the most complex, technological and ritualistic responsibilities. However, in Colombian towns, authorities and officers must organize and exercise their duties agreeing to those rules, which had not necessarily taken into account the special conditions of medium or little size towns. Thus, a sincere thinking over the subject, leaves no more option but to give to the municipalities under special conditions the choice to elect among different organizations and structures that may be reasonable and suitable, according to their needs. In this article the experience of the United States is taken as a reference for in that country, every town, municipality and county, has the legal faculty to choose a form of local government, each one with a specific electoral system, according to the alternatives given by law by each state. 
KEYWORDS: Forms of local government, electoral systems, nomination methods, structural uniformity, local democracy.

TIPO DE ARTíCULO: Reflexión Académica

\section{FORMAS DE GOBIERNO MUNICIPAL Y DE ELECCIONES LOCALES EN ESTADOS UNIDOS Y COLOMBIA}

La descentralización sin especialización y auto-gestión no es más que un arco sin flecha. Después de casi 30 años de descentralización territorial en Colombia cualquiera puede observar que no ha sido suficiente para solucionar nuestra problemática social. Los municipios y los departamentos llevan la misma carga genética de su padre, el nivel central del gobierno: corrupta, poco profesional y lejana de la sociedad. En parte esto es así porque están hechos con el mismo molde, es decir, tienen la misma forma de gobierno y el mismo sistema electoral. Ello ha permitido la agudización de la corrupción y su articulación entre los distintos niveles de gobierno. 
Es muy probable que la estructura aparentemente inerte o neutra de las entidades territoriales sea absolutamente determinante de la calidad de las políticas públicas que deben gestionar y de ello nos ocuparemos en este escrito. Por lo tanto, cabe preguntar: ¿tenemos los ciudadanos derecho a decidir sobre la forma de gobierno y el sistema electoral de las entidades territoriales donde habitamos, en vez de que la Constitución y las leyes definan e impongan estructuras uniformes y rígidas sin considerar las diferencias objetivamente existentes?

\section{La experiencia de los gobiernos locales estadounidenses}

Estados Unidos ha llevado la descentralización hasta un punto extremo ${ }^{1}$. En efecto, los estados han promovido individualmente, a través de sus leyes, la autonomía local de sus gobiernos locales ${ }^{2}$ a través de dos figuras: las facultades de ley general y el poder de 'norma local' o home rule. En el primero de los casos, la ley ha facultado a los gobiernos locales para adaptar a través de un procedimiento democrático formas de gobierno alternativas, cada una con un sistema electoral particular. En el segundo caso, la ley ha permitido a los

\footnotetext{
${ }^{1}$ En el federalismo se encuentra la más pura expresión de la descentralización, pues como consecuencia de la descentralización legislativa se descentraliza el poder ejecutivo y el poder judicial, asignándole poderes reservados a cada uno de los niveles de gobierno que componen la federación.

${ }^{2}$ Por gobierno local se hace referencia a los condados y las municipalidades, tercer y cuarto nivel de gobierno, respectivamente. La organización de los gobiernos locales en Estados Unidos es un tema de reserva estatal. Cada Estado organiza sus gobiernos locales de manera independiente. Esto ha facilitado el surgimiento de un rico laboratorio de gobernabilidad y eficiencia de las instituciones públicas en el nivel local. Sin embargo, las modalidades y procedimientos en materia de organización de los gobiernos locales son similares en todos los estados, debido a la difusión a lo largo de Estados Unidos de las ‘buenas prácticas administrativas' por ligas cívicas y de gobiernos locales tales como la National Civic League o la International City/County Managment Association.
} 
gobiernos locales que legislen ${ }^{3}$ sobre todo aquello que no está prohibido por las leyes federales o estatales, a diferencia de los gobiernos locales de ley general, que sólo pueden legislar sobre aquello que está permitido por la ley ${ }^{4}$. En la práctica, el poder de norma local, generalmente, le permite a los gobiernos locales adaptar, a través de un proceso democrático, una Carta Local (Charter) $)^{5}$. La Carta Local es la Constitución del gobierno local y define, entre otros aspectos, la forma de gobierno y el sistema electoral del gobierno local ${ }^{6}$.

Estas facultades han dado origen a un complejo sistema cooperativo y autogestionado de funciones públicas coordinado desde lo local, pero apoyado económicamente por los estados y la federación a través de programas ${ }^{7}$. Esta maleabilidad y eficiencia de los gobiernos locales, que se encuentra en constante proceso de evolución y retroalimentación, surgió como respuesta a una profunda problemática social que vivieron los estadounidenses al término del siglo XIX y al empezar el siglo XX, en una época recordada como la "era progresista".

\footnotetext{
${ }^{3}$ Generalmente la Constitución o las leyes de cada Estado le dan facultad legislativa a sus gobiernos locales dentro de sus respectivos territorios, en los temas que son de reserva legal de esos gobiernos locales.

${ }^{4}$ Esta regla de origen jurisprudencial es llamada la regla de Dillon, en memoria del juez de Ohio John Dillon, quien en 1.868 sentó este célebre precedente jurisprudencial, que fue acogido ampliamente por las cortes estatales de todos los Estados Unidos.

${ }^{5}$ Ver, por ejemplo la Carta Local de "City of La Junta", Colorado, una ciudad con forma de gobierno de Concejo-Gerente: http://www.ci.la-junta.co.us/charterandcode/CityCharter.pdf

${ }_{6}$ National League of Cities. Local Government Authorities-Home Rule and Dillon's Rule. http://www.nlc.org/about_cities/cities_101/153.aspx.

${ }^{7}$ Las transferencias de dinero de la federación y los estados a los gobiernos locales se hace a través de programas, administrados individualmente por cada departamento, agencia o secretaría. Este sistema se conoce como categorial grants. Los gobiernos locales presentan aplicaciones a los programas que diseñan las dependencias federales o estatales, los cuales tienen un alto grado de especialidad. La oficina que administra el programa puede suspender su ejecución si detecta que el gobierno local no está siendo transparente en su implementación.
} 
La era progresista es registrada por algunos historiadores ${ }^{8}$ entre 1900 y 1918 , entre el gobierno de los presidentes Theodore Roosevelt y Woodrow Wilson. En esta época se impulsaron importantes reformas en la administración pública, como un intento para superar el resquebrajamiento social producido por la industrialización sin regulación. Los monopolios habían promovido la explotación laboral y una corrupción política desenfrenadas, y las instituciones públicas alcanzaron entonces su máximo nivel de ilegitimidad. Ante tal estado de cosas, las principales armas utilizadas por los progresistas para atacar la corrupción y la ineficiencia de las instituciones públicas fueron:

- El sistema electoral no partidario (non partisan elections), las elecciones primarias y la destitución a través del voto (recall elections) para atacar los cacicazgos políticos y la práctica del nepotismo,

- La forma de gobierno de gerente municipal (city manager) para promover gobiernos locales neutrales y eficientes sin sistemas clientelistas ni sesgos políticos, y

- El poder de norma local para sustraer a los gobiernos locales de las malas prácticas administrativas y de las maquinarias políticas estatales.

Como fácilmente puede observarse, el gran objetivo de la era progresista en Estados Unidos fue, y sigue siendo, someter a los políticos al riguroso escrutinio de la opinión pública y a precisos mecanismos de participación democrática.

\footnotetext{
${ }^{8}$ Samuel Elliot Morison y Henry Steele Commager. Historia de los Estados Unidos de América. Segundo Tomo. Fondo de Cultura de México, Traducción de Odón Duran d’Ocón y Faustino Ballvé, México, 1.951, p. 487 y ss. 


\section{Formas de gobierno local}

La forma de gobierno de una comunidad no es solamente una configuración concreta de la burocracia. Es también el resultado de las distintas fuerzas sociales locales luchando por el ejercicio del poder ${ }^{9}$. Por supuesto, un gobierno que demuestra beneficios a los votantes es más estable que un gobierno responsable de haber desatado corrupción e ineficiencia.

En Estados Unidos, anteriormente a la era progresista, existieron dos formas típicas de gobierno local: la de alcalde y la de asamblea general (town meeting). De la era progresista nacieron dos nuevas formas de gobierno local: la de comisión y la de gerente.

1.1. Gobierno de alcalde. Esta forma de gobierno tiene dos variaciones, una de alcalde débil y otra de alcalde fuerte. La primera es la figura tradicional de gobierno local de las pequeñas ciudades estadounidenses, donde el alcalde es sólo un concejal más, preside la corporación y tiene voto sólo en caso de empate en los casos en que el concejo es de número impar. El alcalde débil es generalmente elegido por los concejales y el concejo administra colectivamente el gobierno local. El concejo reúne el poder legislativo y el poder ejecutivo.

\footnotetext{
9 "Las estructuras de gobierno son armas. Ellas establecen una diferencia en cuanto a cómo está gobernada una ciudad y cuáles son sus grupos e intereses más influyentes. Las circunstancias culturales locales determinarán el tipo de estructura deseado por el factor político dominante y el tipo de gobierno que se obtendrá bajo cualquier forma elegida." (Charles Adrian. Governing Urban America. Mc Graw Hill, Nueva York, 1.961, p. 231). Lawrence F. Keller. Forms of Municipal government. Materials Prepared for University Heights Charter Review Commission. Pg. 2. http://www.universityheights.com/downloads/crcformscitygovt.pdf
} 
Por otra parte, la forma de gobierno de alcalde fuerte, que es la forma que tienen todos los municipios colombianos, surge en las grandes ciudades estadounidenses durante el siglo XIX, buscando replicar en el nivel local la tridivisión de poderes y el fuerte liderazgo que ostenta el ejecutivo en los estados y en la federación. Su uso es casi nulo en comunidades medianas y pequeñas.

1.2. Town meeting. Es la forma tradicional de gobierno de los más antiguos asentamientos ingleses en Norteamérica, en la región de Nueva Inglaterra. Es un sistema de verdadera democracia directa, por lo que se usa solamente en ciudades pequeñas. El poder legislativo se encuentra conformado por todos los ciudadanos mayores de edad, reunidos en una reunión anual llamada town meeting. Durante esta reunión los ciudadanos deliberan y votan sobre artículos contenidos en una agenda (warrant). Durante la reunión se elige un moderador que da la palabra y cuenta los votos a favor y en contra, se elige la junta de elegidos (Board of Selectmen) que conforma el poder ejecutivo, se aprueban los impuestos que regirán así como los gastos a ejecutarse, se proponen nuevas normas y se reforman las existentes. Los ciudadanos votan levantando las manos o dando su voto a viva voz, y aplican normas tradicionales que se han transmitido por costumbre en cada comunidad ${ }^{10}$. Debido a la falta de tiempo de los ciudadanos para asistir a la reunión, o a que el pueblo ha aumentado su población, algunas comunidades tienen variaciones representativas. La asamblea

\footnotetext{
10 Ver, por ejemplo, el Manual del pueblo de North Attleborough, Massachusetts, en http://northattleboro.ma.us/clerk/PDF/rtmhandbook.pdf
} 
popularmente elegida puede constar de 60 a 300 ciudadanos, según las regulaciones de cada Estado o comunidad con poder de norma local.

1.3. Gobierno de comisión. Esta forma de gobierno nació en Texas en 1900, cuando un huracán arrasó la ciudad de Galveston ${ }^{11}$. El condado organizó entonces una comisión provisional para atender la emergencia, y este tipo nuevo y especial de gobierno se popularizó por la eficiencia que demostró. Fue ampliamente usado en ciudades pequeñas hasta mitad del siglo $\mathrm{XX}$, pero hoy su uso es reducido. Consiste en una comisión de elección popular que ejerce los poderes legislativo y ejecutivo. Los comisionados se reparten las dependencias administrativas y ejercen colectivamente el poder legislativo. Su impopularidad posterior se debió a que el poder ejecutivo tendía a fraccionarse. Sin embargo, es una forma de gobierno simple y económica, que sigue siendo apropiada para comunidades pequeñas. Su debilidad es corregible si la comisión contrata un administrador o un gerente, lo que genera unidad de mando administrativo.

1.4. Gerente y administrador. La forma de gobierno de gerente-concejo es ampliamente usada por ciudades medianas y pequeñas de los Estados Unidos y en pocos casos por ciudades grandes, como Phoenix, Arizona. Esta forma de gobierno se asemeja a la organización de las empresas privadas. En este tipo de gobierno el poder legislativo se concentra en el concejo y el poder ejecutivo, de carácter más administrativo, es ejercido por un gerente contratado por el concejo.

11 John Edward Weems. Handbook of Texas Online. Galveston Hurricane of 1900. http://www.tshaonline.org/handbook/online/articles/GG/ydg2.html 
Naturalmente, no existe alcalde. El gerente es un administrador público profesional, políticamente neutral, encargado de administrar la municipalidad de manera gerencial. Esta forma de gobierno permite que la municipalidad se administre de acuerdo con criterios de eficiencia y ataca directamente el nepotismo y la corrupción, porque el gerente tiene que demostrar resultados positivos al concejo. De otra manera, el concejo podría despedirlo en cualquier momento ${ }^{12}$.

El administrador es también un administrador público profesional contratado por el concejo o el alcalde, como en el caso de la modalidad de alcalde fuerte con administrador de Los Ángeles, California ${ }^{13}$. Sin embargo, el administrador no tiene los amplios poderes del gerente, tales como nombrar y despedir a los secretarios o cambiar la estructura administrativa del gobierno local. La estabilidad en los gobiernos locales con gerente depende de la transparencia del concejo, que generalmente es elegido por distritos uninominales en elecciones no partidarias para aumentar su responsabilidad política. Los conceptos de gerente y administrador son ampliamente usados en todas las formas de gobierno local estadounidenses ${ }^{14}$.

\footnotetext{
${ }^{12}$ George S. Blair. El Gobierno Local en los Estados Unidos. Traducción de Laura E. Pellegrino. Bibliográfica Omeba, Buenos Aires, 1966, pgs. 222 y ss.

${ }^{13}$ Raphael J. Sonenshine. Los Ángeles: Structure of a City Government. The League of Women Voter of Los Ángeles. 2.006, pgs. 41 y ss. http://www.lwvlosangeles.org/files/Structure_of_a_City.pdf

${ }_{14}$ Council Manager Form of Government. Frequently Asked Questions. Iinternational City/County Managment Association. http://bookstore.icma.org/FreeDocs/Council-Manager\%20FAQ\%20Brochure.pdf
} 


\section{Sistemas electorales para elegir concejo}

El propósito de los distintos instrumentos de elección es lograr la mejor representatividad de los intereses de una comunidad, de tal manera que se encuentre en el cuerpo legislativo local un molde o representación a escala de los problemas y las propuestas de la sociedad ${ }^{15}$. Escoger un sistema electoral implica también definir qué tipo de candidatos y de votantes se espera que participen y la cantidad de responsabilidad política que se genera para los ganadores de las elecciones. Por lo tanto, todo sistema electoral conlleva una combinación concreta de representación ciudadana y de repartición del poder público. El objetivo de un sistema electoral para elegir concejo es conseguir la fórmula más equilibrada entre representación y gobernabilidad. Muchas variables pueden afectar el comportamiento de los distintos sistemas electorales en sociedades determinadas. Debido a estas razones, el equilibrio entre gobernabilidad y representación se puede conseguir diseñando el sistema electoral adecuado según las características concretas de cada comunidad. En palabras de Dieter Nohlen, "no hay ningún sistema ideal o teóricamente superior que pueda pasar sin más el test de la comprobación histórico-empírica. (...) El mejor sistema es el que mejor se adapta a estas circunstancias cambiantes" ${ }^{16}$.

2.1. Sistemas electorales partidarios y no partidarios: Los sistemas electorales pueden ser partidarios o no partidarios (non partisans). En los partidarios

\footnotetext{
15 Austin Ranney y Willmoore Kendall. La Democracia y el Sistema de los Partidos Políticos en Estados Unidos. Traducción de Julio Barrancos Money. Bibliográfica Argentina. Buenos Aires, 1.958, pg. 82 y 83.

${ }^{16}$ Dieter Nohlen. Sistemas Electorales en su Contexto. Instituto de Investigaciones Jurídicas. UNAM, México D. F., 2.008 , pg. 5 .
} 
corresponde a los partidos nominar candidatos, como en Colombia. En los no partidarios se prohíbe a los partidos políticos proponer candidatos y se prohíbe a los candidatos identificarse con partidos políticos en el tarjetón electoral. Las elecciones no partidarias "se fundaban en la teoría de que los gobiernos locales eran corporaciones en lugar de cuerpos políticos y que las cuestiones del gobierno local eran de naturaleza administrativa más que política, por lo que los funcionarios debían semejarse más a hombres de negocios que a políticos" ${ }^{17}$.

Los candidatos en los sistemas no partidarios son de carácter independiente y se inscriben con peticiones firmadas por una cantidad de 25 a 500 ciudadanos, dependiendo del número de habitantes de la comunidad. Las candidaturas independientes, que fomentan la cultura del voto de opinión, permiten a los ganadores de las elecciones administrar imparcialmente y sin ataduras clientelistas. En las elecciones no partidarias se vive una fuerte competencia entre personalidades políticas de carácter cívico, en vez de fomentarse rivalidades ideológicas o alianzas temporales de partidos. 'Los problemas sociales no son de carácter demócrata o de carácter republicano, son problemas que necesitan políticas públicas neutrales y eficientes', usualmente argumentan quienes defienden las elecciones no partidarias. A la luz de las elecciones partidarias, no tiene fundamento que un partido político llegue al poder para gobernar sólo como le parece al partido y sólo para los seguidores del partido, cuando las políticas públicas deben ser de carácter neutral y favorecer a todos los que están bajo las

\footnotetext{
${ }^{17}$ George S. Blair. El gobierno local en los Estados Unidos, op. cit., pg. 143.
} 
mismas condiciones por igual. En otras palabras, las elecciones no partidarias buscan promover gobiernos de ciudadanos ideológicamente neutrales, proponiendo nuevos esquemas de gobernabilidad.

La ciudad de Los Ángeles y el Estado de Nebraska eligen sus cuerpos legislativos y sus líderes ejecutivos mediante elecciones no partidarias y la ciudad de Nueva York ha intentado implantar ese sistema por iniciativa de sus dos últimos alcaldes (Roy Giulliani y Michael Bloomberg), reforma que en distintas oportunidades no ha superado las instancias democráticas. La gran mayoría de las ciudades pequeñas a lo largo de los Estados Unidos practican elecciones no partidarias. Las elecciones no partidarias también se utilizan para elegir personas a cargos uninominales de elección popular.

2.2. Métodos de nominación: En sistemas no partidarios los candidatos se inscriben con peticiones firmadas por ciudadanos. La nominación por petición democratiza la participación en política porque permite que cualquier ciudadano pueda aspirar a puestos de elección popular. La competitividad de las elecciones hace que los ciudadanos sin seria vocación política se abstengan de inscribirse y que los candidatos sin experiencia pierdan fácilmente o se retiren antes de la votación.

En sistemas partidarios es más común que los candidatos se nominen a través de elecciones primarias, que pueden ser cerradas $\mathrm{o}$ abiertas (si es reservada 
solamente para miembros del partido o no), o pueden nominarse por caucus, es decir, por decisión de las directivas locales del partido. Las elecciones primarias permiten que las bases electorales designen los candidatos del partido y en elecciones por caucus el líder o líderes del partido imponen los candidatos a la base electoral. En el caso de Colombia, la nominación es por caucus.

2.3. Métodos de elección: Existen tres métodos de elección: por distrito único, por distritos uninominales y por distritos plurinominales ${ }^{18}$.

2.3.1. Distrito único (at-large). En este caso los candidatos provienen de cualquier parte de la ciudad, haciendo muy difícil la representación pareja de todas las zonas, pues sólo quedan representados los sectores ricos o políticamente activos de la comunidad. Los electores tienen derecho a tantos votos como curules haya en el concejo. Este método favorece las mayorías únicamente, pero hay cuatro métodos que permiten aumentar la proporcionalidad: el voto limitado, que limita la cantidad de votos del elector, buscando disminuir el voto en bloque ${ }^{19}$ de las mayorías; el voto transferible, que le permite al elector numerar los candidatos, asignándole mayor fuerza a los primeros que a los últimos; el voto acumulativo, que permite al elector depositar todos sus votos a favor del mismo candidato, $y$, finalmente, el voto por listas de partido, que le permite a las directivas del partido hacer una

\footnotetext{
${ }^{18}$ City Council Elections Methods. The Center for Voting and Democracy.

http://www.fairvote.org/media/documents/City_Council_Manual.pdf

${ }^{19}$ Voto por todos los candidatos del mismo partido.
} 
lista de candidatos para que de acuerdo con una cifra repartidora se distribuyan las curules. Este último método, que es el usado en Colombia a nivel nacional y local, ha demostrado disminuir la responsabilidad política de los ganadores de las elecciones, dado que la base electoral no participa en la formación de la lista ${ }^{20}$.

2.3.2. Distritos uninominales. En este caso el territorio del municipio se divide en tantos distritos como curules haya en el concejo, cuidando que todos tengan el mismo número de habitantes. Cada distrito elige sólo un concejal y los candidatos deben ser habitantes del respectivo distrito. Cada elector sólo tiene un voto. Con este método electoral se logra una pareja representación de los diversos sectores del municipio y se pueden erigir distritos minoritarios o de interés especial para favorecer un grupo poblacional o un sector particular del municipio que requiera de atención especial. Este método electoral también permite elevar la competitividad de las elecciones y aumenta la responsabilidad política y la 'identificabilidad' de los ganadores de las elecciones. A menudo se combinan con elecciones por distrito único para mantener la visión global de la comunidad y evitar la sectorización excesiva de las políticas públicas. Al ser más competitivo, este sistema mejora la calidad de los elegidos y su mayor visibilidad. Así se

${ }^{20}$ City Council Elections Methods. The Center for Voting and Democracy. Op. cit., pg. 18. 
generan mecanismos de responsabilidad política o accountability, esto es, rendición de cuentas a los electores ${ }^{21}$.

2.3.3. Distritos plurinominales. Son usados para elegir la asamblea en la forma de gobierno de asamblea representativa (representative town meeting). El territorio se divide en varios distritos y en cada uno se pueden elegir dos o más concejales. A medida que se elijan más concejales de un distrito plurinominal el método gana en proporcionalidad, pero pierde en visibilidad y responsabilidad política, asimilándose al sistema de distrito único. Por el contrario, entre menos concejales se elijan de un distrito plurinominal, más se asimila el método al de distritos uninominales ${ }^{22}$.

\section{La realidad colombiana}

En Colombia el esquema constitucional de descentralización territorial ha fijado una estructura uniforme para el gobierno de todas las entidades territoriales. Este principio de uniformidad es remanente de la igualdad ante la ley $\mathrm{y}$, por consiguiente, ante la administración, triunfante en la Revolución Francesa ${ }^{23}$. La Constitución de 1991 establece para los municipios y los departamentos una

\footnotetext{
21 Augusto Hernández Becerra. Del departamento del siglo XIX al del siglo XXI, en "Crisis y Futuro de los Departamentos en Colombia”. Bogotá, Universidad Externado de Colombia y Konrad Adenauer Stiftung, 2003, pg. 216.

${ }^{22}$ Para conocer más a fondo la administración pública local de los Estados Unidos consultar: Augusto Hernández Vidal, "Gobierno Local en los Estados Unidos e Ideas para una Reforma Municipal”, Tesis de Grado. 2010. Biblioteca de la Universidad Santo Tomás.

${ }^{23}$ Augusto Hernández Becerra. Objetivos Inéditos de la Categorización Municipal, en "Problemática de los Municipios Pequeños en Colombia ¿Supresión o Reforma?”. Bogotá, Universidad Externado de Colombia y Konrad Adenauer Stiftung, 2003, pg. 111.
} 
forma de gobierno de ejecutivo fuerte-corporación débil. La ley, por su parte, ha fijado para los municipios y departamentos un sistema electoral de distrito único con listas de partido cuyos candidatos son inscritos por las directivas del partido. Estas reglas impuestas a los habitantes de las entidades territoriales de manera general y uniforme, no han demostrado promover transparencia y eficiencia. En lugar de ello se han amañado para volver cotidiana la corrupción y la mala administración, que la sociedad contempla con perplejidad, desprovista de herramientas jurídicas para impulsar cambios y correctivos a las instituciones de sus gobiernos locales.

La lección que se puede aprender de la era progresista en Estados Unidos es la misma que Andrew Jackson dejó a los demócratas: el pueblo es el mejor protector de la fe pública. A Simón Bolívar, por su parte, se le atribuye la siguiente frase: "Tan sólo el pueblo conoce su bien y es dueño de su suerte. Pero no un poderoso, ni un partido, ni una fracción"24. Para empezar a resolver la problemática social desde las instituciones públicas locales, primero es necesario crear espacios de amplio debate popular, que en Estados Unidos son llamados town meetings y en Colombia de antaño se conocieron como cabildo abierto ${ }^{25}$. En estos espacios públicos de deliberación los ciudadanos manifiestan sus inconformidades y los académicos y especialistas pueden canalizarlas en soluciones definitivas 0 experimentales de gobierno, que a su vez retroalimentarán y cristalizarán instituciones públicas más especializadas a las necesidades reales, más

\footnotetext{
${ }^{24}$ Pedestal del monumento al Libertador, Plaza de Bolívar, Bogotá.

${ }^{25}$ El cabildo abierto en realidad se encontraba limitado a los habitantes destacados o con tierras de la sociedad.
} 
transparentes y eficientes. Estas deliberaciones pueden incluso girar alrededor de la conveniencia de implementar una u otra forma de gobierno, o uno u otro sistema electoral en las entidades territoriales. ¿Por qué es razonable discutir democrática y racionalmente otras formas de gobierno y de elección? Porque las organizaciones gubernamentales y los modos de elegir son los principales elementos estructurales de la institucionalidad que tienen impacto sobre lo que hacen las entidades territoriales, es decir, sobre los bienes y servicios públicos que deben proveer a la comunidad. Esto es evidente si se tiene en cuenta que las diversas formas de gobierno y de sistemas electorales que pueden aplicarse a los gobiernos locales favorecen distintos esquemas de ejercicio del poder político y de participación popular.

La imposición general y centralizada de una forma de gobierno y de un sistema electoral comunes a las entidades territoriales, desconoce la soberanía popular, la autonomía de las entidades territoriales, el pluralismo y, por tanto, no es democrática. La organización de las entidades territoriales colombianas en tales condiciones resulta siendo ilegítima, porque sus habitantes nunca participaron en su creación. La democracia es más tangible cuando es posible para los ciudadanos participar en la transformación estructural de sus instituciones públicas locales. Pero nunca hemos celebrado un contrato social a nivel local en Colombia. 


\section{Formas de gobierno alternativas para las entidades territoriales}

La forma de gobierno de ejecutivo fuerte en las entidades territoriales colombianas ha fomentado una rivalidad malsana entre el ejecutivo y la corporación tanto en municipios como en departamentos. La manera más común de viabilizar la 'gobernabilidad' ha venido a ser la corrupción pactada o tolerada, siempre tras bastidores, claro está. Las mayorías que controlan la corporación le exigen al alcalde o al gobernador beneficios personales concretos a cambio de que sus proyectos se aprueben. Incluso se hacen de antemano pactos políticos con los candidatos, comprometiendo el intercambio de votos por favores ${ }^{26}$. Así se construyen, desde las campañas políticas, las mal denominadas "coaliciones". De esta manera las políticas públicas impulsadas por el ejecutivo quedan sujetas a los intereses clientelistas de los miembros de la corporación, provocando inevitablemente una administración torpe y sin fortaleza gerencial. Como consecuencia de estas prácticas, muchas entidades territoriales colombianas se han convertido en máquinas burocráticas con poca disposición hacia la innovación o la inclusión social.

Por otra parte, la uniformidad estructural de las entidades territoriales ignora las circunstancias especiales propias de cada entidad territorial que deben tenerse en cuenta para lograr una mejor administración. Las características poblacionales y

\footnotetext{
${ }^{26}$ Ver: Francisco Leal y Andrés Dávila. Clientelismo: el sistema político y su expresión regional. Tercer Mundo, Bogotá. 1990.
} 
geográficas son indicadores de la forma de gobierno y el sistema electoral que serían apropiadas para cada entidad territorial. Las comunidades rurales, por ejemplo, pueden recibir mejor una forma de gobierno de corporación fuerte, donde los acuerdos y ordenanzas se aprueban y se ejecutan por la misma corporación, dado que los intereses de estas comunidades están enfocadas principalmente hacia el desarrollo de las formas de explotación equitativa de la tierra y el cuidado del medio ambiente, para lo cual es indispensable la participación del campesinado. Una corporación fuerte es más representativa, simple y económica que una forma de ejecutivo fuerte-corporación débil, por lo que funciona mejor en comunidades rurales ${ }^{27}$. Por su parte, las ciudades en crecimiento y las grandes ciudades necesitan formas de gobierno más gerenciales, profesionales y estructuradas, dadas las numerosas y complejas problemáticas que deben manejar. La necesidad de toma decisiones inteligentes y su ejecución práctica y eficiente, requiere, en estas ciudades, de administraciones entrenadas, eficaces y transparentes bajo la unidad de mando que puede dar un Gerente o un alcalde con el apoyo de administradores en áreas estratégicas.

\footnotetext{
${ }^{27}$ El municipio colombiano colonial tuvo un gobierno de corporación fuerte, rasgo particular que ha sido difícil abandonar por los actuales concejos municipales. Cfr. Augusto Hernández Becerra. Debilidad Institucional del Municipio y Crisis Institucional del Modelo de gobierno Municipal, en Revista ZERO, Universidad Externado de Colombia, Bogotá, $\mathrm{N}^{\circ}$ 19, segundo semestre 2007, p. 20 y ss.
} 


\section{Sistemas electorales alternativos para las entidades territoriales}

Todas las entidades territoriales colombianas tienen un sistema electoral de distrito único con listas de partido inscritas por directivas de partido ${ }^{28}$. Este sistema electoral permite que los concejos municipales y las asambleas departamentales se compongan de ciudadanos con baja responsabilidad política, porque los candidatos que nomina el partido no son necesariamente candidatos que la sociedad apruebe o siquiera conozca. En la mayoría de los casos los candidatos que integran las listas de partido son personas cercanas al partido, o que le conviene candidatizar para conservar el statu quo político. La participación de los ciudadanos en los partidos políticos es muy pasiva, ya que lo único que se les permite es elegir entre los candidatos que presentan las directivas del partido. Este sistema dificulta también la posibilidad de que ciudadanos no pertenecientes a la clase política tradicional resulten electos para cargos de elección popular, entorpeciendo el derecho político de los candidatos cívicos a ser elegidos. Estas son características de un sistema electoral elitista, poco inclinado hacia la innovación y francamente antidemocrático, que podría ser contrarrestado con la práctica de elecciones primarias y de elecciones no partidarias.

Con un sistema de distrito único ni los concejos municipales ni las asambleas departamentales pueden representar equitativamente los habitantes de la entidad

\footnotetext{
${ }^{28}$ La consulta popular es una posibilidad no practicada por los partidos políticos colombianos, en el caso de elección de corporaciones públicas, alcaldes y gobernadores.
} 
territorial, especialmente si estos son muy numerosos, si viven en condiciones sociales muy desiguales, si la circunscripción electoral es muy extensa o si no pertenecen a ningún partido, hipótesis que se presentan en la gran mayoría de los casos. El sistema de distrito único sólo es representativo en comunidades pequeñas y cuyos habitantes están en condiciones sociales muy similares, ya que en comunidades con estas características no es trascendental la residencia o el estatus social de los ganadores de las elecciones para lograr una justa representación. En comunidades con complejos problemas sociales y que comprenden áreas extensas, un sistema de distrito único es discriminador y poco representativo, porque los ganadores de las elecciones populares normalmente provienen de clases sociales privilegiadas a los que no les tocan los problemas de los otros sectores de la sociedad ${ }^{29}$. Especialmente ocurre este fenómeno si los candidatos del partido no son producto de una consulta popular. A través de distritos uninominales o plurinominales se podría garantizar que los ganadores de las elecciones representen intereses locales concretos, legítimos y prioritarios ${ }^{30}$.

El actual sistema electoral permite que la clase política tradicional se mantenga estable a pesar de los escándalos públicos que continuamente protagonizan, pues no permite que haya candidatos con ideas alternativas que podrían y deberían aspirar con éxito a un cargo de elección popular.

\footnotetext{
${ }^{29}$ Este fenómeno, ciertamente, puede ser contrarrestado por la existencia de partidos populares.

${ }^{30}$ Augusto Hernández Becerra. Del departamento del siglo XIX al del siglo XXI. Op. cit., pg. 216.
} 


\section{Una propuesta}

Nuestro sistema jurídico debería permitir a cada entidad territorial tomar la iniciativa de adaptar sus instituciones a las necesidades particulares de su entorno político y social, contando en todo ese proceso con la participación propositiva de sus ciudadanos y el apoyo técnico de economistas, politólogos y planificadores expertos en administración pública. De esta manera las entidades territoriales podrían adaptarse a los cambios sociales y políticos que naturalmente ocurren en todas las comunidades. Dichas iniciativas tomarían en cuenta factores tales como:

- El papel de los ciudadanos como legitimadores de sus instituciones públicas,

- El estudio científico de la administración pública municipal y departamental,

- La gobernabilidad de las entidades territoriales,

- La responsabilidad y autogestión de las entidades territoriales,

- La eficiencia de las entidades territoriales,

- La adecuación de las funciones de gobierno a las necesidades reales de la comunidad,

- La construcción colectiva de lo público,

- La comprensión y el interés ciudadano por lo público, y

- En términos generales, la paz y la democracia.

La Constitución prevé la posibilidad de que las entidades territoriales se administren y organicen de manera diversa a través de la figura de la 
categorización (artículos 302 y 320), posibilidad que no ha sido debidamente explorada por el legislador, siendo que esa posibilidad existe desde 1945 para los municipios $^{31}$, y desde 1991 para los departamentos. Sin embargo, sería conveniente ir más allá de lo previsto en la Constitución, porque de lo que se trata es de convertir a la entidad territorial en protagonista de la decisión sobre la forma de gobierno y el sistema electoral que mejor se acomode a sus condiciones concretas. Para posibilitar esta idea hace falta una habilitación constitucional que permita a las entidades territoriales iniciar procesos de 'asamblea constituyente local', en un proceso democrático y participativo. En estas condiciones se permitiría a municipios y departamentos, por iniciativa de las autoridades de la entidad territorial o por iniciativa popular de sus habitantes, escoger entre una de las formas de gobierno y uno de los sistemas electorales que la ley proponga por vía general. La decisión final de forma de gobierno y de sistema electoral tendría que ser aprobada, en un referendo, por la mayoría de los votantes de la entidad territorial.

\section{Reflexión final}

Otorgar a la gente el poder de opinar y decidir sobre la estructura y organización de sus entidades territoriales sería una decisión hito en la historia política de un país de tradición política centralista como Colombia. Como consecuencia de esa

\footnotetext{
${ }^{31}$ Augusto Hernández Becerra. Objetivos Inéditos de la Categorización Municipal. Op. cit., pg. 115.
} 
medida, los gobiernos locales tendrían la opción de volverse realmente locales y podrían sustraerse notablemente de la lógica centralista del poder político, creando cierta tendencia hacia el federalismo, dentro del marco de la ley que regule este proceso. De esta manera la administración estaría más cerca de solucionar las necesidades cada vez mayores de una sociedad en desarrollo que requiere de instituciones públicas más transparentes y eficientes.

\section{REFERENCIAS}

Adrian, Ch. (1961). Governing Urban America. Nueva York: .Mc Graw Hill.

Blair, G. (1966). El Gobierno Local en los Estados Unidos. Traducción de Laura E. Pellegrino. Buenos Aires: Bibliográfica Omeba.

Hernández, A. (2003). Del departamento del siglo XIX al del siglo XXI, en "Crisis y Futuro de los Departamentos en Colombia". Bogotá: Universidad Externado de Colombia y Konrad Adenauer Stiftung, pg. 216.

Hernández, A. (2003). Objetivos Inéditos de la Categorización Municipal, "Problemática de los Municipios Pequeños en Colombia ¿Supresión o Reforma?". Bogotá: Universidad Externado de Colombia y Konrad Adenauer Stiftung, pg. 111. 
Hernández, A, (2010). Gobierno Local en los Estados Unidos e Ideas para una Reforma Municipal, Tesis de Grado no publicada. Universidad Santo Tomás.

Leal, F., \& Dávila, A. (1990). Clientelismo: el sistema político y su expresión regional. Bogotá: Tercer Mundo.

Morrison, S. \& Commager, H. (1951 Historia de los Estados Unidos de América. (Segundo Tomo). México: Fondo de Cultura de México, Traducción de Odón Duran d'Ocón y Faustino Ballvé.

Nohlen, D. (2008). Sistemas Electorales en su Contexto. México D. F: Instituto de Investigaciones Jurídicas UNAM,.pg. 5.

Ranney, A., \& Kendall, W. (1958). La Democracia y el Sistema de los Partidos Políticos en Estados Unidos. Traducción de Julio Barrancos Money. Buenos Aires:. Bibliográfica Argentina.

Hernández, A. (2007). Debilidad Institucional del Municipio y Crisis Institucional del Modelo de gobierno Municipal, Revista ZERO, № 19. 
Sonenshine, R. (2006). Los Ángeles: Structure of a City Government. The League of Women Voter of Los Ángeles. http://www.Iwvlosangeles.org/files/Structure of a City.pdf 